\title{
Pten and p53 Converge on c-Myc to Control Differentiation, Self-renewal, and Transformation of Normal and Neoplastic Stem Cells in Glioblastoma
}

\author{
H. Zheng, ${ }^{* w}$ H. Ying, ${ }^{* w}$ H. Yan, ${ }^{*}$ A.C. Kimmelman, ${ }^{* \dagger}$ D.J. Hiller, ${ }^{*}$ A.-J. Chen, ${ }^{*}$ S.R. PerRy, ${ }^{*}$

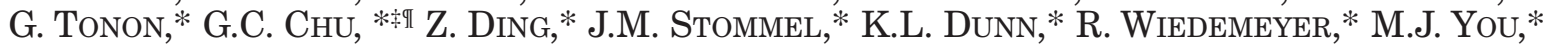

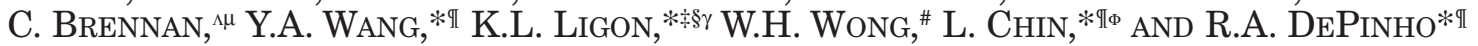 \\ *Department of Medical Oncology, "Center for Applied Cancer Science, Belfer Foundation Institute for Innovative \\ Cancer Science, ${ }^{\gamma}$ Center for Molecular Oncologic Pathology, Dana-Farber Cancer Institute and Harvard Medical \\ School, ${ }^{\dagger}$ Harvard Radiation Oncology Program, ${ }^{*}$ Department of Pathology, ${ }^{\circledR}$ Division of Neuropathology, ${ }^{\oplus}$ Department \\ of Dermatology, Brigham and Women's Hospital, Harvard Medical School, Boston, Massachusetts; "Department of \\ Statistics, Stanford University, Stanford, California; ${ }^{\lambda}$ Department of Neurosurgery, Memorial Sloan-Kettering Cancer \\ Center, New York; ${ }^{\mu}$ Department of Neurosurgery, Weill-Cornell Medical College, New York, New York
}

\begin{abstract}
Glioblastoma (GBM) is a highly lethal primary brain cancer with hallmark features of diffuse invasion, intense apoptosis resistance and florid necrosis, robust angiogenesis, and an immature profile with developmental plasticity. In the course of assessing the developmental consequences of central nervous system (CNS)-specific deletion of p53 and Pten, we observed a penetrant acute-onset malignant glioma phenotype with striking clinical, pathological, and molecular resemblance to primary GBM in humans. This primary, as opposed to secondary, GBM presentation in the mouse prompted genetic analysis of human primary GBM samples that revealed combined $p 53$ and Pten mutations as the most common tumor suppressor defects in primary GBM. On the mechanistic level, the "multiforme" histopathological presentation and immature differentiation marker profile of the murine tumors motivated transcriptomic promoter-binding element and functional studies of neural stem cells (NSCs), which revealed that dual, but not singular, inactivation of $p 53$ and Pten promotes cellular c-Myc activation. This increased c-Myc activity is associated not only with impaired differentiation, enhanced self-renewal capacity of NSCs, and tumor-initiating cells (TICs), but also with maintenance of TIC tumorigenic potential. Together, these murine studies have provided a highly faithful model of primary GBM, revealed a common tumor suppressor mutational pattern in human disease, and established c-Myc as a key component of p53 and Pten cooperative actions in the regulation of normal and malignant stem/progenitor cell differentiation, self-renewal, and tumorigenic potential.
\end{abstract}

Malignant glioma, the most common intrinsic brain tumor in adults, is nearly uniformly fatal (Furnari et al. 2007). The most advanced form of malignant glioma, glioblastoma multiforme (GBM), carries a median survival of only 9-12 months, a statistical fact that has changed little despite innumerable clinical trials during the past several decades (Reardon et al. 2006). Several hallmark biological properties of malignant gliomas are the likely basis for the intractable nature of GBM, including an intense apoptosis resistance, rampant genome instability, and a propensity to disseminate early and widely throughout the brain - rendering surgical resection noncurable.

The identification of clonogenic TICs with stem-like properties across many different tumor types has revealed a level of plasticity in human cancer cells previously unrecognized (Bonnet and Dick 1997; Al-Hajj et al. 2003; Singh et al. 2004b; Taylor et al. 2005; O’Brien et al. 2007; RicciVitiani et al. 2007). These clonogenic cells, regardless of their actual cell(s) of origin, constitute a reservoir of selfsustaining cells with the exclusive ability to self-renew and

${ }^{\psi}$ These authors contributed equally to this work. maintain the tumor. Moreover, the TIC or "cancer stem cell" hypothesis is predicated on the idea that not all glioma cells have equal proliferative potential and that cells with the greatest ability to proliferate and form new tumors have phenotypic and functional properties similar to those of NSCs (Singh et al. 2004a; Clarke et al. 2006). During the past several years, multiple reports have established the existence of TICs in human GBM that are endowed with superior tumorigenic potential in orthotopic models and possess self-renewal and multilineage cell differentiation potential-properties that are eerily reminiscent of NSCs (Singh et al. 2004b; Bao et al. 2006; Lee et al. 2006;).

The phenotypic similarities of GBM TICs and NSCs have fueled speculation that neural stem/progenitor cell compartments may be the preferred cell of origin for GBM, although the dedifferentiation and transformation of more differentiated glial cells have been demonstrated experimentally (Bachoo et al. 2002). The latter findings have implications for the cancer stem cell hypothesis because it suggests that although at any given time certain cells may have TIC properties, the ready capacity for dedifferentiation raises the possibility that "TIC differentiated progeny" could reacquire an immature tumorigenic state. If so, this plasticity would have implications for the treat- 
ment of cancer because it would imply that complete eradication of the TIC compartment would not lead to durable cures, as is widely assumed, and that such eradication would need to be complemented with differentiationinducing agents. In this light, it stands to reason that an improved understanding of the pathways governing processes of differentiation along the NSC-glial axis and, by extension, the TIC-progeny axis, may prove instrumental in guiding more effective drug development efforts.

During the past 20 years, molecular and human genetic studies have revealed obligate pathways involved in GBM pathogenesis, and principal among these are the RBp16/p18-CDK4/6, p53-MDM2-ARF, and PI3K/PTEN/ AKT pathways (Zhu and Parada 2002; Furnari et al. 2007). A wealth of studies has established a central role for the phosphoinositol-3 kinase (PI3K) pathway in the pathogenesis of glioma on the genomic, genetic, and epigenetic levels (Furnari et al. 2007). Historically, p53 inactivation has been considered to be a more classical genetic lesion in low-grade astrocytomas that invariably evolve into secondary GBM, whereas primary GBMs are thought to more commonly sustain loss of the CDKN2A locus, which contains both cyclin-dependent kinase inhibitor 2A (Ink4a) and an alternate open reading frame (ARF), a negative regulator of the p53 pathway (Watanabe et al. 1996; Kleihues and Ohgaki 1999). On the other hand, recent populationbased studies have observed $p 53$ mutations in primary GBM, raising the possibility that $p 53$ might also serve as a tumor suppressor in the primary GBM subtype (Ohgaki et al. 2004; Fukushima et al. 2006). Moreover, our recent effort on unbiased global copy-number analysis of human primary GBM has identified both Pten and $p 53$ deletion by genome topography scanning (Wiedemeyer et al. 2008).

In the course of assessing the effect of singular or combined impact of $p 53$ and Pten deficiencies on CNS development and gliomagenesis, our studies have generated a faithful model of primary GBM, prompted resequencing studies that uncovered a high frequency of $p 53$ mutations in human primary GBM (a lesion previously considered to be more classical for secondary GBM), and revealed cooperative interactions of $p 53$ and Pten deficiencies in the regulation of glial cell differentiation. Along these lines, we show that conditional p53 and Pten inactivation in neural stem cells and resultant tumors promotes normal and malignant cell self-renewal, impedes their differentiation, and sustains potent malignant potential. An integrated genomic and functional analysis indicates that these two tumor suppressors converge on the regulation of Myc to control these cellular processes.

\section{RESULTS}

\section{Combined Loss of $p 53$ and Pten in the Mouse Brain Leads to Highly Penetrant Malignant Glioma Development with Striking Similarity to Human Primary GBM}

To genetically address the role and genetic interactions of p53 and Pten in CNS development, NSC biology, and glioma pathogenesis, we used a constitutive hGFAP-Cre transgenic line (Zhuo et al. 2001) to target deletion of p53 alone or in combination with Pten in the NSC compartment and its derivative CNS lineages. Early and broad CNS hGFAP-Cre activity was evidenced by Cre-mediated activation of the Rosa26-LacZ reporter in the forebrain and hindbrain as well as in germinal zones of the embryonic brain (E13.5). Consequently, Cre-mediated recombination is present throughout neural stem/progenitor cells and mature lineages of the adult CNS (data not shown). Correspondingly, hGFAP-Cre Pten ${ }^{l o x} /$ ox mice display the early postnatal lethal phenotype of hydrocephalus and macrocephaly, as previously reported in the Pten-deficient brain (Backman et al. 2001; Kwon et al. 2001; data not shown). Because broad CNS deletion in mice homozygous for Pten $^{l o x / l o x}$ resulted in an early postnatal lethal phenotype of hydrocephalus and macrocephaly, the Pten ${ }^{\text {lox/+ }}$ genotype was used for glioma modeling in this study.

By 15-40 weeks of age, $42 / 57(73 \%)$ mice in the hGFAP-Cre $p 53^{\text {lox/lox }}$ Pten ${ }^{\text {lox } /+}$ cohort presented with acuteonset neurological symptoms including seizure, ataxia, and/or paralysis, resulting in death with median survival of 28 weeks (Fig. 1A). Necropsy and histopathological analyses revealed that all 42 neurologically symptomatic mice harbored malignant gliomas that, by World Health Organization (WHO) 2007 classification criteria (Louis et al. 2007), were determined to be anaplastic astrocytomas (WHO Grade III, $n=36,64 \%$ ) or GBM (WHO Grade IV, $n=14,25 \%$ ) (Fig. 1B,C). All tumors exhibited marked cellular pleomorphism and diffuse infiltrative spread in a single-cell manner with formation of secondary structures of Sherer, including perineuronal and perivascular satellitosis and subpial collections in the cerebral cortex (Fig. 1D). The tumors classified as WHO Grade IV (GBM) displayed the defining histopathological features of necrosis with pseudopalisading and, less frequently, necrosis combined with microvascular proliferation (Fig. 1D). These tumors had a striking resemblance to the human disease with increased mitotic indices as indicated by $\mathrm{Ki}-67$ staining and extensive immunohistochemical staining for the human glioma markers GFAP and Nestin (Fig. 1C). The remaining $15(15 / 57)$ hGFAP-Cre p53 lox/lox Pten $^{\text {lox } /+}$ mice succumbed to non-CNS-derived tumors, either sarcomas or breast adenocarcinomas that result from $h G F A P-C r e$ activity outside of the CNS, as documented by low-level Cre-mediated deletion of the floxed alleles in non-CNS tissues (data not shown).

In addition to these end point analyses, we also conducted a serial histopathological analysis of 15 mice before the onset of neurological symptoms. Surprisingly, no mice were found to have developed tumors analogous to human low-grade gliomas. Instead, 8/15 mice exhibited high-grade (at least WHO Grade III) pathology including some very small lesions composed of highly anaplastic cells with features of nuclear atypia, multinucleated tumor cells, and/or dense cellularity (Fig. 1E); thus, even early lesions show highly aggressive malignant features from the outset. Together, the rapidly progressive clinical course and pathologic WHO Grade IV classification in a significant number of mice indicate that the hGFAP-Cre $p 53^{\text {lox } / l o x} \mathrm{Pten}^{\text {lox/+ }}$ model most faithfully recapitulates the clinical and pathological features of the human primary GBM subtype. 
A

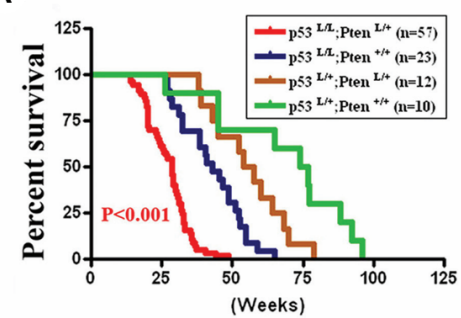

C

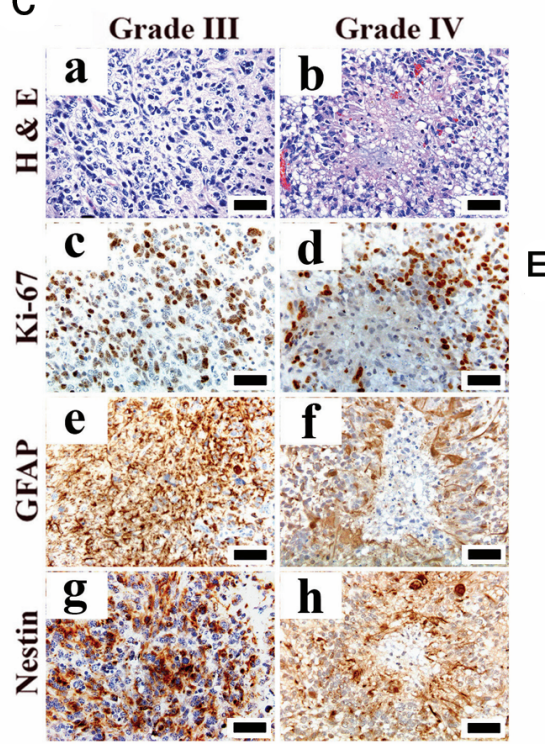

B

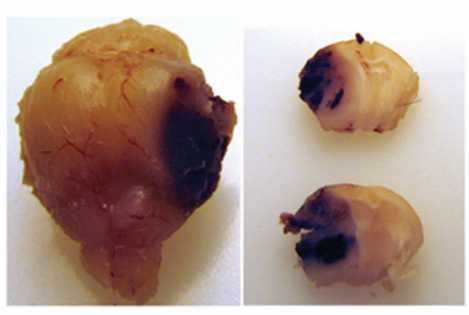

D
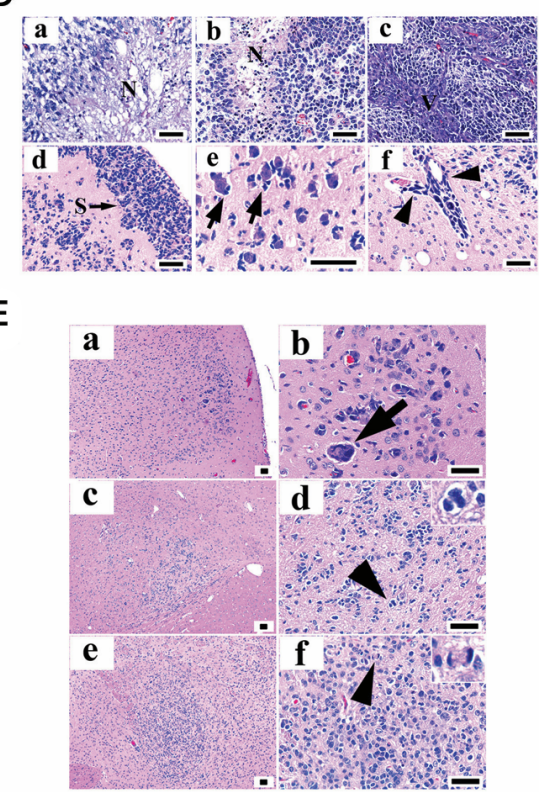

Figure 1. Inactivation of $p 53$ and Pten induces primary malignant gliomas. (A) Kaplan-Meier tumor-free survival curves for mice of indicated genotypes as a function of weeks. $(B)$ Representative of malignant gliomas after biopsy. $(C)$ H\&E (hematoxylin and eosin) histology and immunohistochemical staining of sections of WHO grade-III and -IV malignant gliomas from $h G F A P-C r e ; P 53^{\text {lox } / l o x} ;$ Pten ${ }^{\text {lox } /+}$ mice. (D) Histological features of glioblastomas observed in $h$ GFAP-Cre;P5 $3^{\text {loxlox}} ;$ Pten ${ }^{l o x /+}$ mouse brains. "N" represents areas of the palisading with regional necrosis in the tumors $(a, b)$; "V" regions suggest microvascular proliferation $(c)$; "S," subpial spread $(d)$; arrows in $e$ point to perineuronal satellitosis and arrowheads in $f$ point to perivascular satellitosis. $(E)$ Small emerging tumors in asymptomatic mice show highly malignant histological features. Arrow in $b$ points to multinucleated giant cell; arrowheads in $d$ and $f$ indicate mitotic figures amid the tumor cells. Bars, $50 \mu \mathrm{m}$. (Reprinted from Zheng et al. 2008 [C Nature Publishing Group].)

\section{Acquired Molecular Profile of hGFAP-Cre p53 $3^{\text {lox/lox }}$ Pten $^{\text {lox/+}}$ Gliomas Mirrors Classical Alterations of the Human Disease}

To further assess the human relevance of the $h G F A P-C r e$ p5 $3^{\text {lox/lox }}$ Pten $^{\text {lox/+ }}$ model, we examined the acquired molecular profile of the murine high-grade gliomas. In human high-grade glioma, loss of heterozygosity ( $\mathrm{LOH})$ of chromosome $10 \mathrm{q}$ encompassing the Pten locus is one of the most frequent genomic alterations, occurring in 60-70\% of cases (Louis 2006; Furnari et al. 2007). Consistent with the critical need to extinguish Pten in advanced gliomas, the high-grade murine $h$ GFAP-Cre $p 53^{\text {lox/lox }} \mathrm{Pten}^{\text {lox/+ }}$ glioma cells showed consistent loss of anti-Pten immunoreactivity as the result of losing the remaining wild-type Pten allele (Fig. 2A,B). Along with complete loss of Pten expression within the tumor cells, examination of key PI3K signaling surrogates revealed robust activation of AKT and S6kinase in all samples tested (Fig. 2C). Moreover, the enhanced cyclin D1 expression and high level of vascular endothelial growth factor (VEGF) observed in human high-grade disease are also present in these murine malignant gliomas, whereas much lower levels were detected in the adjacent normal brain tissues (Fig. 2C).

The highly penetrant GBM phenotype in the absence of an engineered receptor tyrosine kinase (RTK) allele was notable given the frequent amplification and overexpression of epidermal growth factor receptor (EGFR) in human primary GBM (Libermann et al. 1985) and the effectiveness of mutant activated RTK alleles in murine glioma models (Fomchenko and Holland 2006). At the same time, all human primary GBMs show coactivation of multiple RTKs, commonly EGFR and platelet-derived growth factor receptor- $\alpha$ (PDGFR $\alpha$ ), and many of these activated RTKs do not show genomic or genetic alterations yet function cooperatively to maintain tumor cell viability (Stommel et al. 2007). Consistent with this notion, the robust anti-PDGFR $\alpha$ signal was detected in murine glioma cells relative to that in adjacent normal cells (Fig. 2D). Most tumors concurrently showed strong 


\section{ZHENG ET AL.}

A

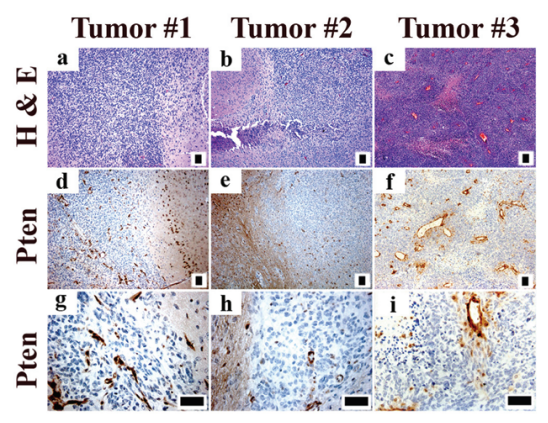

C

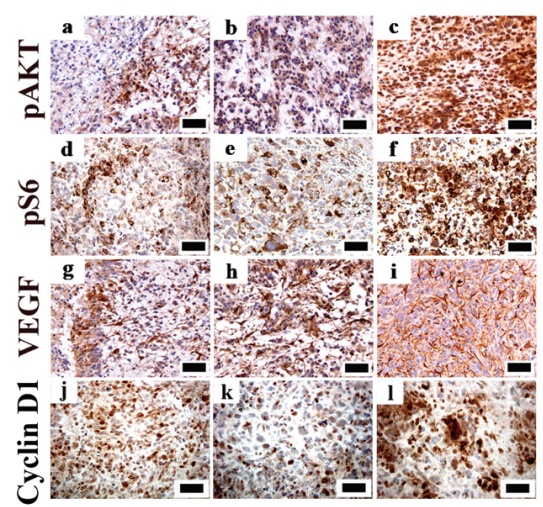

B
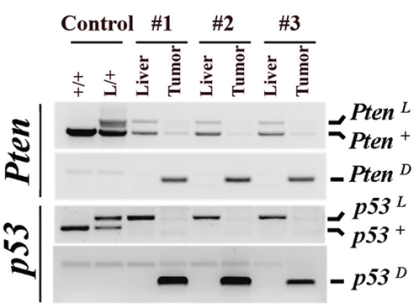

D
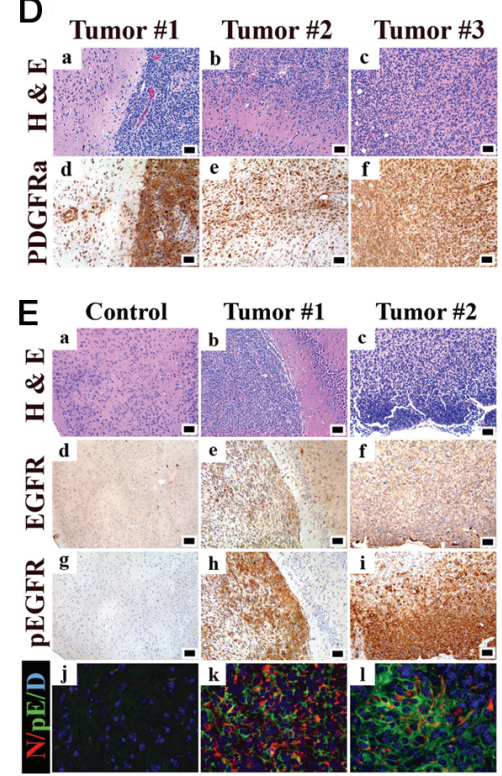

Figure 2. hGFAP-Cre; $P 53^{l o x} / 0 x ; P_{t e n}^{l o x /+}$ gliomas mirror key features of human malignant gliomas. $(A)$ Pten expression is completely extinguished in tumor cells. $(B)$ Wild-type Pten allele is lost in glioma cells. Genomic DNA isolated from liver tissues and brain tumor cells were subjected to polymerase chain reaction (PCR)-based assays for genotyping Pten and p53 alleles. Note that "+" designates the Pten wild-type allele, " $L$ " the conditional allele, and " $D$ " the inactivated form of the conditional allele after Cre-mediated recombination. $(C)$ Immunohistochemical staining of murine glioma sections with antibodies against activated p-AKT, p-S6 kinase, VEGF, and cyclin D1. Bars, $50 \mu \mathrm{m}$. (D) PDGFR $\alpha$ is specifically overexpressed in tumor cells as compared to normal brain cells. $(E)$ EGFR is activated in malignant glioma cells. Ajacent sections from control normal brain and two independent high-grade gliomas were subjected to H\&E, immunohistochemical staining for EGFR, and phospho-EGFR (pEGFR). Bars, $50 \mu \mathrm{m}$. (Reprinted from Zheng et al. 2008 [C Nature Publishing Group].)

regional activation of EGFR, which is less conspicuous in the relatively solid tumor centers and more robust in the subpial regions, as well as the more infiltrative fronts with high proliferative activity (Fig. 2E). Of interest, a fraction of strong phospho-EGFR tumor cells was intensely positive for Nestin, a finding that may relate to the prominent biological role of EGF signaling in less mature CNS cells including NSCs (Bachoo et al. 2002; Doetsch et al. 2002).

\section{Pten and p53 Are Frequently Mutated in Human Primary GBM}

The striking clinical and histopathological resemblance of the $\mathrm{p} 53 /$ Pten model with human primary GBM prompted analysis of $\mathrm{p} 53$ mutational status in the primary GBM subtype in humans. To this end, we performed resequencing and copy-number analyses of Pten and p53 genes in a collection of 35 clinically annotated and pathologically verified human primary GBM samples; 26 of these samples were previously profiled by array-CGH (comparative genome hybridization) (Wiedemeyer et al. 2008). Ten out of $35(29 \%)$ tumors registered prototypical "hot spot" tumor-relevant p53 mutations, and 14/35 (40\%) tumors possessed inactivating Pten mutations including missense mutations, insertions, deletions, and splicing mutations. Among the 10 samples harboring p53 mutations, six also showed Pten mutations or homozygous deletion. In addition, all five samples with wild-type Pten copy-number and sequence status were free of $p 53$ mutations. Together, these genomic studies indicate that combined inactivation of p53 and Pten is an unexpectedly frequent genetic profile in human primary GBM.

\section{Stem/Progenitor Cell Features}

A classical feature of human primary GBM is its high degree of intertumoral and intratumoral morphological and lineage heterogeneity, hence the moniker glioblastoma "multiforme." These morphological characteristics were readily evident in hGFAP-Cre p53 lox/lox $\mathrm{Pten}^{\text {lox/+ }}$ gliomas (Fig. 3A). The basis for the morphological variability is not known and may relate, among many possibilities, to the maintenance or acquisition of a developmental state with multipotency and/or differentiation plas- 
A

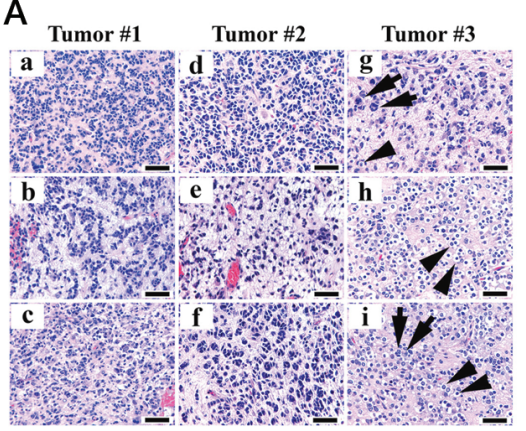

B Tumor\#1 Tumor \#2 Tumor\#3

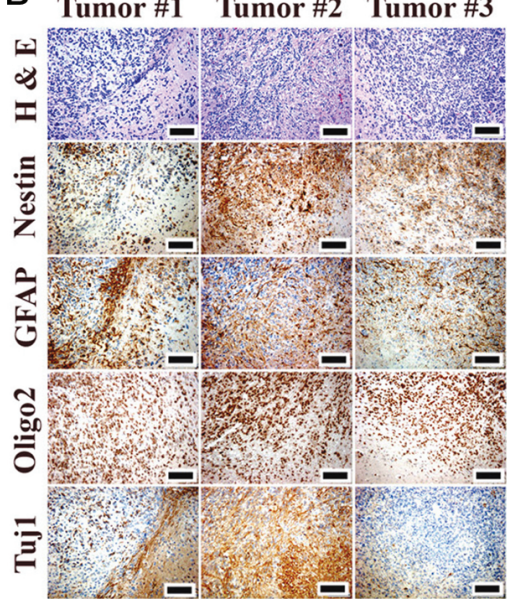

C

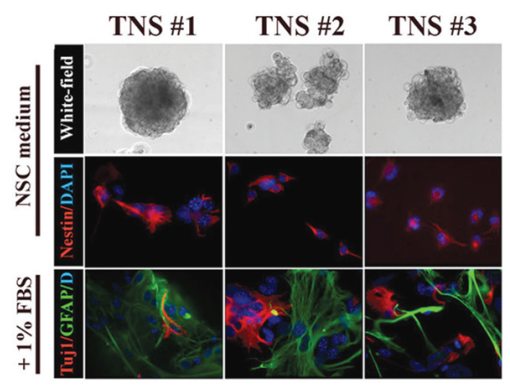

D H \& E GFAP

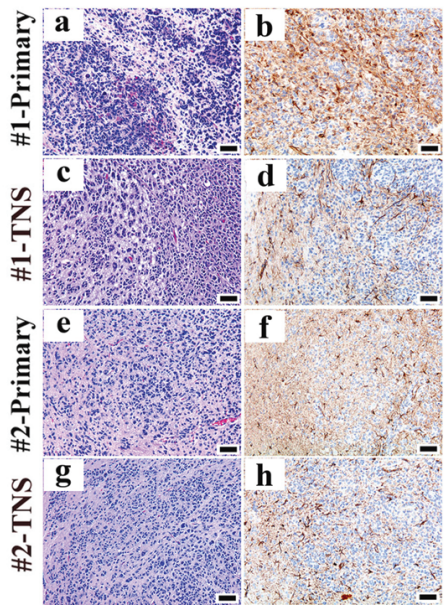

Figure 3. $h$ GFAP-Cre;P53 $3^{\text {lox/lox}} ;$ Pten $^{\text {lox } /+}$ murine malignant gliomas present stem/progenitor features. $(A)$ Malignant gliomas possess high intertumoral and intratumoral histological heterogeneity. $(a-c, d-f$, and $g-i)$ Three different regions of tumor 1,2 , and 3 , respectively. Note the mixed conspicuously fibrillated matrix of astrocytoma and "fried egg" appearance of oligodendroglioma features in tumor $3(g-i)$; arrows designate astrocytoma cells and arrowheads indicate oligodendroglioma cells. (B) Sections from three independent high-grade gliomas were subjected to H\&E and immunohistochemical staining with antibodies against Nestin, GFAP, Olig-2, and Tuj1. Note the lack of Tuj 1 staining in the no. 3 tumor cells. (C) TNS lines isolated from independent $h G F A P-C r e ; P 53^{\text {lox } / l o x} ; P_{\text {Pten }}^{\text {lox } /+}$ gliomas were cultured in NSC medium or differentiation medium with 1\% FBS and immunostained for lineage markers Nestin, GFAP, and Tuj1 as indicated. $(D)$ Secondary tumors generated from orthotopic injection of TNS cells recapitulate the pathological features of primary tumors. Bars, $50 \mu \mathrm{m}$.

ticity resulting from cancer-relevant genetic and epigenetic alterations. Along these lines, it is intriguing that occasional tumors $(5 / 50)$ presented with both astrocytic and oligodendroglial histopathological features within a single tumor (Fig. 3A), suggesting the existence of transformed cells in these tumors that retain the capacity to differentiate into different cell types. In line with this speculation, all murine tumors expressed stem or lineage progenitor markers commonly observed in human gliomas, including the stem/progenitor marker Nestin $(\mathrm{N})$, the astrocytic lineage marker GFAP (G), and the oligodendroglial progenitor marker Olig2 (O) (Fig. 3B) (Ligon et al. 2007), and were negative for mature neuronal and oligodendrocyte markers NeuN and MBP (data not shown). Intriguingly, the murine tumors could be further classified into two subgroups on the basis of neuronal lineage marker Tuj 1 expression that is present in about $80 \%$ of tumors and completely absent in the remainder, reminiscent of the proneural, proliferative, and mesenchymal subclasses found in the human disease (Phillips et al. 2006). In light of the developmental hierarchy and increasing evidence of the stem-like TICs residing within gliomas, these subsets may reflect the TIC differentiation status, in which $\mathrm{N}^{+} \mathrm{G}^{+} \mathrm{O}^{+} \mathrm{T}^{+}$tumors originate from TICs resembling NSCs or early progenitors and $\mathrm{N}^{+} \mathrm{G}^{+} \mathrm{O}^{+} \mathrm{T}^{-}$tumors from TICs resembling lineage-committed progenitors. Alternatively, the propensity for a proneural phenotype may reflect acquired alterations in the TICs that control lineage development.

This stem/progenitor profile is in accord with the ability of all tumors tested to readily generate tumor neurospheres (TNSs) from single-cell suspensions of freshly resected and dissociated malignant murine glioma tissues with (1) very robust NSC/progenitor marker Nestin expression, (2) limited capacity to differentiate into astrocytic and neuronal lineages upon exposure to differentiation agents (Fig. 3C), and (3) strong tumor-initiating potential in which as few as 500 cells orthotopically injected into the forebrain of SCID (severe combined immunodeficiency disease) mice could readily generate secondary tumors histopathologically resembling their primary tumors (Fig. 3D). These data provide further evidence for the existence of cells displaying the tentatively defined properties of TICs within murine gliomas. 


\section{ZHENG ET AL.}

\section{Pten and 553 Inactivation Impedes NSC Differentiation in a Myc-dependent Manner}

Prompted by the immature marker profile of the murine tumor and the likelihood that NSCs/progenitor cells may be the preferred cell of origin for GBM (Sanai et al. 2005; Zhu et al. 2005; Gilbertson and Gutmann 2007), we hypothesized that the gliomagenic impact of Pten and $p 53$ deficiencies might be executed in part by affecting processes of $\mathrm{NSC} /$ progenitor cell self-renewal and/or differentiation. To test this hypothesis, we characterized the cellular and molecular properties of primary NSC cultures as a function of $p 53$ and/or Pten status. Consistent with previous reports that Pten and p53 negatively regulate the proliferation and selfrenewal capacity of NSCs (GilPerotin et al. 2006; Groszer et al. 2006; Meletis et al. 2006), NSCs null for either Pten or p53 showed a modestly increased proliferative rate and neurosphere formation capacity after each passage. However, combined loss of $\mathrm{p} 53$ and Pten resulted in a marked increase in NSC proliferation and self-renewal capacity (Fig. 4A,B), pointing to strong cooperative interactions of these tumor suppressors in the regulation of NSC biology.

The enhanced NSC self-renewal, coupled with the aforementioned varied histological tumor presentations, raised the possibility that combined p53 and Pten deficiency might impede the differentiation potential of the NSC/progenitor cultures. When continuously cultured in serum-free media supplemented with EGF and basic fibroblast growth factor (bFGF), NSCs of various genotypes showed similar morphology and robust expression of the NSC/progenitor marker Nestin and minimal expression of differentiated lineage markers, such as GFAP (glial fibrillary acidic protein) for astrocytes, $\mathrm{O} 4$ for oligodendrocytes, and Tuj1 for neuronal lineages (Fig. 4C). However, differentiation differences emerged among the
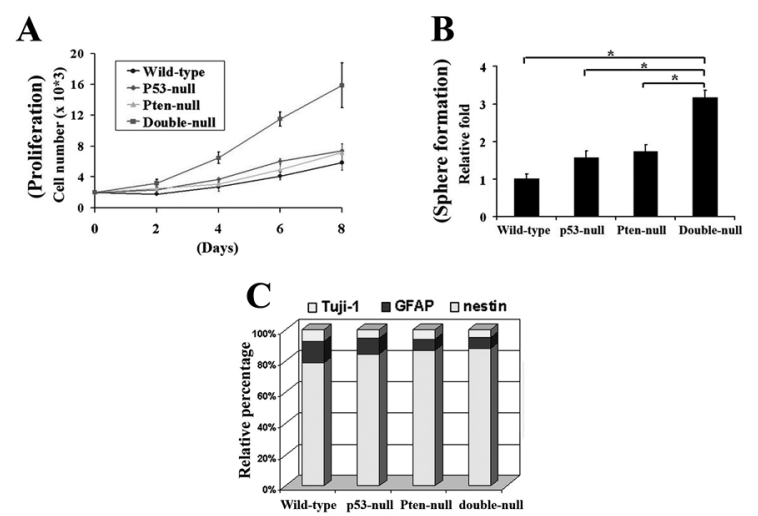

Figure 4. Mutant NSCs present enhanced proliferation and selfrenewal. (A) Proliferation of $p 53 /$ Pten double-null NSCs is enhanced as compared with wild-type and singly null NSCs $(n=$ $3)$. (B) The number of multipotent neurospheres formed by p53/Pten double-null NSCs in culture is significantly increased as compared with neurospheres of wild-type or singly null NSC controls $(* P<0.001 ; n=3)$. (C) Quantification histograms indicate that percentages of different lineage markers staining positive cells in wild-type and mutant NSCs are comparable when cultured in NSC medium $(n=3)$. Nestin, NSC/progenitor marker; GFAP, astrocyte lineage marker; Tuj1, neuronal lineage marker. genotypes. Specifically, upon exposure to differentiationinducing medium containing 1\% fetal bovine serum (FBS), the majority of wild-type and single-null NSCs assumed a flattened morphology, lost Nestin staining and differentiated into either GFAP-positive astrocytes, Tuji1positive neurons, or O4-positive oligodendrocyte (Fig. 5A). In contrast, NSCs doubly null for both $p 53$ and Pten failed to respond fully to these differentiation cues and retained stem-cell-like morphology and lineage-marker staining after 7 days under differentiation-induction conditions. Immunofluorescence staining analysis confirmed that approximately $50-60 \%$ of cells retained Nestin expression, whereas the remaining cells showed a range of lineage marker profiles. Importantly, NSCs singly null for either $p 53$ or Pten did not exhibit such resistance to differentiation induction. Accordingly, inhibition of AKT by Triciribine (Yang et al. 2004) readily reversed differentiation resistance in the double-null NSCs (Fig. 5B). These findings point to the cooperative impact of $p 53$ and Pten deficiency in preserving the stem-cell-like state in the face of potent differentiation cues.

To understand the molecular basis for the impaired differentiation capacity of double-null NSCs, we performed transcriptome comparison of p53-null and p53/Pten double-null NSCs following exposure to a differentiation inducer. At day 1 of induction, 410 genes exhibited significant differential expression. Gene ontology (GO) analysis of these differentially expressed genes revealed enrichment of cell cycle and neural development functions, consistent with the observed differentiation defect. Intriguingly, promoter analysis on the 410 differentially expressed genes identified E2F and Myc motifs as two of the most enriched promoter-binding elements with 1.7- and 1.4-fold increases, respectively. The identification of c-Myc as a potential driving force in our system was encouraging given its established roles in cell cycle progression and apoptosis (Pelengaris et al. 2002; Patel et al. 2004) and the accumulating evidence of its functions as a key regulator of stem cell self-renewal and differentiation during development and oncogenic processes (Coppola and Cole 1986; Cartwright et al. 2005; Takahashi and Yamanaka 2006;

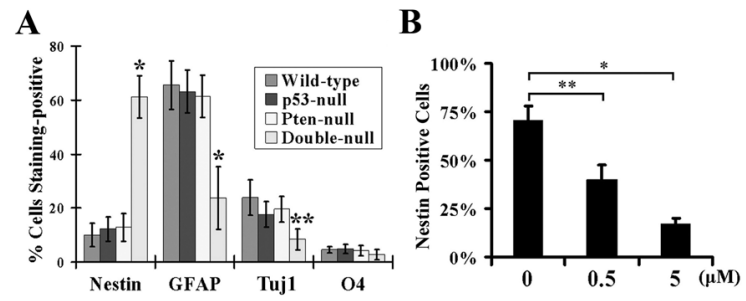

Figure 5. $p 53$ and Pten doubly inactivated NSCs are defective of their differentiation capacity. (A) Multilineage differentiation induced by $1 \%$ FBS was impaired in double-null NSCs as compared with wild-type and singly null NSCs $(* P<0.005 ; * * P<$ $0.05 ; n=3)$. (B) Inhibition of the PI3K/AKT pathway sensitizes p53/Pten double-null NSCs to differentiation. p53/Pten doublenull NSCs cultured in 1\% FBS in the absence or presence of AKT inhibitor (Triciribine) for 7 days were subjected to immunofluorescent staining with antibodies against Nestin and GFAP $(* * P<$ $\left.0.05 ;{ }^{*} P<0.001 ; n=3\right)$. 
A

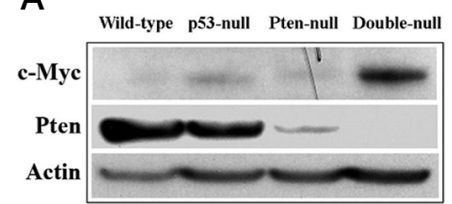

B
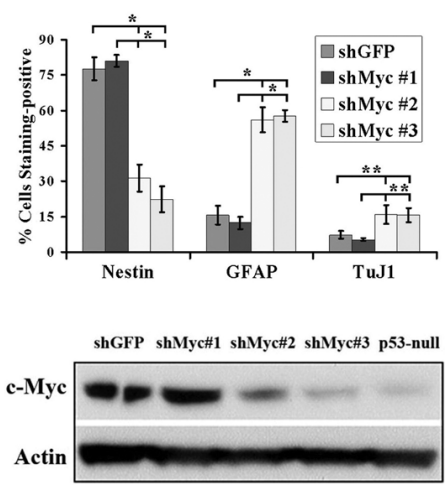

Figure 6. $p 53$ and Pten coordinately regulate cellular c-Myc protein levels and NSC differentiation. $(A)$ Combined inactivation of $p 53$ and Pten in NSCs stimulates c-Myc protein expression. $(B)$ Knockdown of c-Myc expression in p53/Pten double-null NSCs restores differentiation capacity in response to 1\% FBS. (Upper panel) Histograms show percentage of indicated cells immunopositive for different lineage markers $(* P<0.005 ; * * P<0.05 ; n$ $=3$ ). (Lower panel) Western blot showing c-Myc protein expression of double-null NSCs infected with indicated lenti-shRNA. Note that both lenti-shRNA against GFP and shMyc no. 1 functioned as controls. (Reprinted from Zheng et al. 2008 [C Nature Publishing Group].)

Ben-Porath et al. 2008; Chang et al. 2008). Consistent with this, western blot analysis shows a substantial increase in cMyc protein levels in the $p 53$ and Pten double-null NSCs and only marginally increased c-Myc expression in single p53-null or Pten-null NSCs when compared to wild-type cells (Fig. 6A). The prominent enrichment of Myc promoter-binding motifs in this unbiased integrated transcriptomic/promoter analysis, coupled with the impaired differentiation phenotype, raised the possibility that $p 53$ and Pten act coordinately to regulate c-Myc levels, which in turn control NSC self-renewal and differentiation.

To test this hypothesis, we examined the impact of cMyc knockdown on the differentiation potential of p53/Pten double-null NSCs. We observed that two independent short hairpin RNAs (shRNAs) (nos. 2 and 3) against c-Myc, which attenuated c-Myc expression in double-null NSCs to the levels comparable to those levels seen in p53-null NSCs, largely restored the NSC differentiation capacity upon exposure to differentiation-inducing medium containing 1\% FBS (Fig. 6B). Conversely, enforced c-Myc expression in p53-null NSCs, but not empty vector controls, repressed their differentiation potential, and the cells retained expression of the stem/progenitor markers of Nestin and Sox 2 upon differentiation induction (data not shown). Together, these data indicate that concomitant loss of both $p 53$ and Pten tumor suppressors impedes differentiation and enhances renewal of NSCs in a c-Myc-dependent manner.

\section{c-Myc Regulates Tumor Neurosphere Differentiation, Renewal, and Tumorigenic Potential}

The potent and pleiotropic activities of c-Myc requires tight control of its expression in developing and adult tissues, and its deregulation contributes to the genesis of various cancer types including gliomas (Nesbit et al. 1999; Bredel et al. 2005; Ben-Porath et al. 2008). Our finding that c-Myc impacts on NSC/progenitor cell biology prompted us to further assess the relevance of c-Myc on the malignant potential of murine-glioma-derived TICs; such TICs are enriched in TNS cultures. First, consistent with the importance of PI3K pathway activation, AKT inhibitor treatment of the murine glioma TNS cells strongly attenuated c-Myc protein expression and promoted their differentiation (Fig. 7A,B). Correspondingly, c-Myc knockdown using two independent shRNAs markedly reduced TNS cell proliferation and self-renewal capacity (Fig. 8A,B). Moreover, upon exposure to differentiation-induction conditions, c-Myc knockdown strongly sensitized the murine-glioma-derived TNS cells to differentiate, whereas control TNS cells retained an NSC/progenitor marker Nestin-positive phenotype (Fig. $8 \mathrm{C})$. With respect to tumorigenic potential, intracranial injection of vector-transduced murine-derived TNS cells resulted in lethal infiltrating gliomas that resemble primary tumor pathology in 10/10 mice within 1 month (Fig. 8D), whereas $9 / 10$ mice implanted with c-Myc knockdown TNSs continue to survive for several months. Together, these data support the view that c-Myc has a key

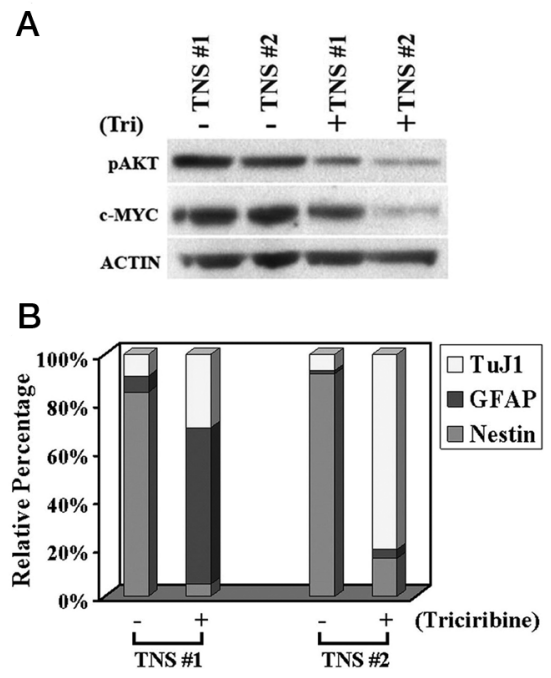

Figure 7. Inhibition of the AKT pathway induces TNS cell differentiation. (A) Inhibition of the AKT pathway in TNS cells with Triciribine attenuates their cellular c-Myc expression. $(B)$ Inhibition of AKT pathway with Triciribine induces TNS cell differentiation $(n=3)$. Two independent TNS lines (TNS 1 and TNS 2) were cultured in $1 \%$ FBS in the absence or presence of Triciribine $(5 \mu \mathrm{M})$ for 7 days before being subjected to immunostaining with antibodies against Nestin, GFAP, and Tuj1. 
A

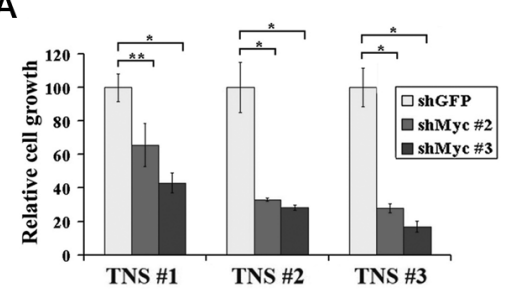

C

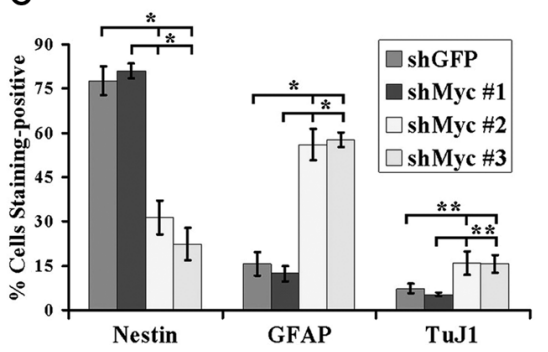

$\mathrm{B}$

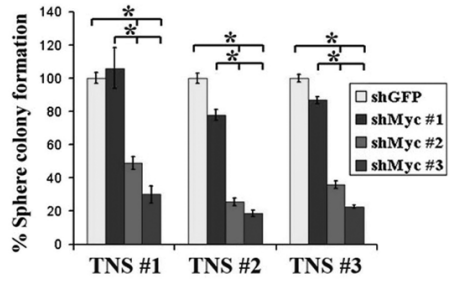

D

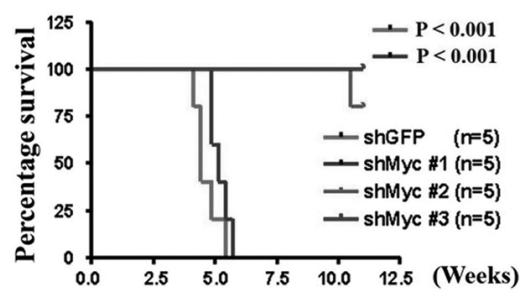

Figure 8. Attenuated c-Myc expression restores $h G F A P-C r e ; P 53^{l o x} / l o x ; P t e n^{l o x} /+$ TNS differentiation potential and reduces their tumorigenic potential. $(A)$ Knockdown of c-Myc expression reduces TNS cell growth. TNS cells infected with indicated lenti-shRNA virus were cultured in NSC medium and cell proliferation was measured 6 days after plating $\left({ }^{*} P<0.01 ; * P<0.001 ; n=3\right)$. (B) Reduction of c-Myc expression in TNS cells reduces their self-renewal potential assessed by sphere formation $(* P<0.001, n=3)$. (C) shRNAmediated reduction of c-Myc expression in TNS cells sensitizes cells to differentiation stimuli $(* P<0.005 ; * * P<0.05 ; n=3)$. For all graphs, values represent mean \pm S.D. from at lease three experiments. $(D)$ Knockdown of c-Myc expression represses TNS tumorigenic potency after orthotopic transplantation into SCID mice. (Reprinted from Zheng et al. 2008 [C Nature Publishing Group].)

role in impaired differentiation and potent tumorigenic potential of $p 53 /$ Pten-null TNS cells.

\section{CONCLUSION}

In the present study, we demonstrate that dual inactivation of $p 53$ and Pten produces a primary GBM phenotype that shares many of the molecular and histopathological features of the human disease. Our genomic analysis of p53 and Pten in human primary GBM revealed that they are the two most commonly mutated tumor suppressors, thereby supporting the relevance of the hGFAP-Cre; $p 53^{\text {lox/lox}} ;$ Pten $^{\text {lox } /+}$ glioma model and the utility of genetically engineered mouse models to guide the analysis of human cancer genomics. More importantly, this mousemodeling effort demonstrates how this approach affords an experimental framework to understand on a mechanistic level how specific genetic alterations cooperate to affect malignant transformation. Along these lines, using an integrative genomic and functional analysis, we have demonstrated c-Myc as a focal point for the cooperative actions of combined $p 53$ and Pten deficiencies, consistent with studies showing that $\mathrm{p} 53$ can repress $c-M y c$ transcription through directly binding to the $c-M y c$ promoter (Ho et al. 2005), and an activated PI3K can promote mammalian target of rapamycin (mTor)-dependent c-Myc translation as well as glucogen synthase kinase-3 $\beta$ (GSK$3 \beta$ )-induced c-Myc protein stability (Sears et al. 2000; Gera et al. 2004).

p53 and PI3K/Pten pathways are universally altered in human GBM (Furnari et al. 2007). Loss of $p 53$ in mice has been shown to elevate self-renewal of adult NSCs (GilPerotin et al. 2006; Meletis et al. 2006), whereas murine brain-specific inactivation of Pten has been shown to cause aberrant NSC and astrocyte proliferation and brain overgrowth during development (Backman et al. 2001; Kwon et al. 2001; Groszer et al. 2006), suggesting their relevance in the regulation of NSC biology. The role of Pten in stem cell biology gains further significance from recent hematopoietic studies establishing that acute somatic deletion of Pten leads to initial expansion but ultimate depletion of hematopoietic stem cells (HSCs) (Yilmaz et al. 2006; Zhang et al. 2006). This progressive depletion suggests either the activation of exhaustion pathways causing stem cell exhaustion and/or the absence of renewal-promoting pathways. The former may stem from decreased FoxO family activity, which has been shown to deplete long-term HSC reserves via loss of quiescence control, increased apoptosis, and increased intracellular reactive oxygen species (ROS) levels (Tothova et al. 2007). In the context of the findings of our current study, the latter may relate to insufficient activation of a cMyc-driven stem cell renewal program.

The prominence of the c-Myc proto-oncogene in our model system was of particular interest because it represents one of a handful of factors known to both interfere with normal stem cell biology and enable malignant transformation (Shachaf et al. 2004; Wong et al. 2008). Our experimental data in premalignant NSCs are in line with the perspective that sustained c-Myc expression blocks cell differentiation and promotes self-renewal in embryonic stem cells (ESCs) and cancers (Lassman et al. 2004; Shachaf et al. 2004; Ben-Porath et al. 2008; Wong et al. 2008), a notion reinforced by the recent findings of c-Myc as one of the four factors sufficient to reprogram mouse or human fibroblasts into embryonic stem (ES) cells (Takahashi and Yamanaka 2006; Jaenisch and Young 2008). This function of c-Myc may relate to its ability to 
globally reprogram and maintain an active chromatin state (Knoepfler et al. 2006). The recent discovery by Wong et al. (2008) and Ben-Porath et al. (2008) that an ES-cell-like gene expression signature is enriched in poorly differentiated aggressive human tumors including malignant gliomas further supports the idea that c-Myc functions to promote tumorigenesis by inappropriately altering the machinery controlling the cellular differentiation state. Our finding that p53 and Pten pathways converge on cMyc and its downstream targets therefore provides a rational molecular explanation and model for the defective differential traits of the double-null NSCs as well as the TNSs derived from the resultant tumors (Fig. 9).

The remarkable similarities shared between normal stem cells and cancer cells, such as self-renewal and differentiation potential, have motivated efforts to compare these two cellular states (Reya et al. 2001). Identification of TICs with stem-like properties in diverse human cancers including GBM represents an important conceptual advancement in cancer biology, with important implications for the diagnosis and treatment of cancers (Clarke and Fuller 2006; Lobo et al. 2007). Like normal NSCs, glioma TICs, which appear to constitute a reservoir of self-sustaining cells with potent tumorigenic potential, reside in perivascular niches that maintain the stem-like properties of these cancer cells (Calabrese et al. 2007). Moreover, when orthotopically transplanted into the brains of immunodeficient mice, the isolated TICs form tumors that are histologically identical to the original tumor, suggesting that the TICs retain the capacity to generate all cell types found in the parent tumor and can fully recapitulate the neoplastic phenotype in vivo (Singh et al. 2004b; Lee et al. 2006). However, unlike normal NSCs that readily differentiate along a developmental hierarchy into lineage-restricted progenitors and their differentiated progenies (Gage 2000; Alvarez-Buylla et al. 2001; Temple 2001), malignant glioma cells, which display phenotypic plasticity mirroring normal NSCs, often lack the terminal differentiation traits possessed by their normal counterparts. In support of this notion, the murine $p 53 /$ Pten doubly null TNS cells show desensitization toward differentiation cues. Forced restoration of the differentiation capacity in the TNS cells through either AKT inhibitor treatment or shRNA-mediated c-Myc ablation greatly diminishes their tumorigenic potential. The dramatic attenuation of self-renewal and tumorigenic potential upon down-regulation of c-Myc in murine TNSs, combined with in silico evidence of its dysregulation in human primary GBM, encourages the identification and testing of novel or existing combinational therapies targeting differentiation-promoting pathways including cMyc in the treatment of primary GBM.

\section{ACKNOWLEDGMENTS}

We thank A. Berns for providing $p 53^{L}$ mice; S. Zhou and S. Jiang for excellent mouse husbandry and care; R.T. Bronson for helpful discussion on pathology analysis; K. Montgomery for discussion on sequencing; and Y.-H. Xiao, B. Feng, and J. Zhang for bioinformatic help. H.Z. was supported by the Helen Hay Whitney Foundation. H.Y. is a recipient of the Marsha Mae Moeslein fellowship from the American Brain Tumor Association. A.C.K. is a recipient of the Leonard B. Holman Research Pathway fellowship. Z.D is supported by the Damon Runyon Cancer Research Foundation. J.M.S is supported by a Ruth L. Kirschstein National Research Service Award fellowship. R.W. is supported by a Mildred Scheel Fellowship (Deutsche Kreb-

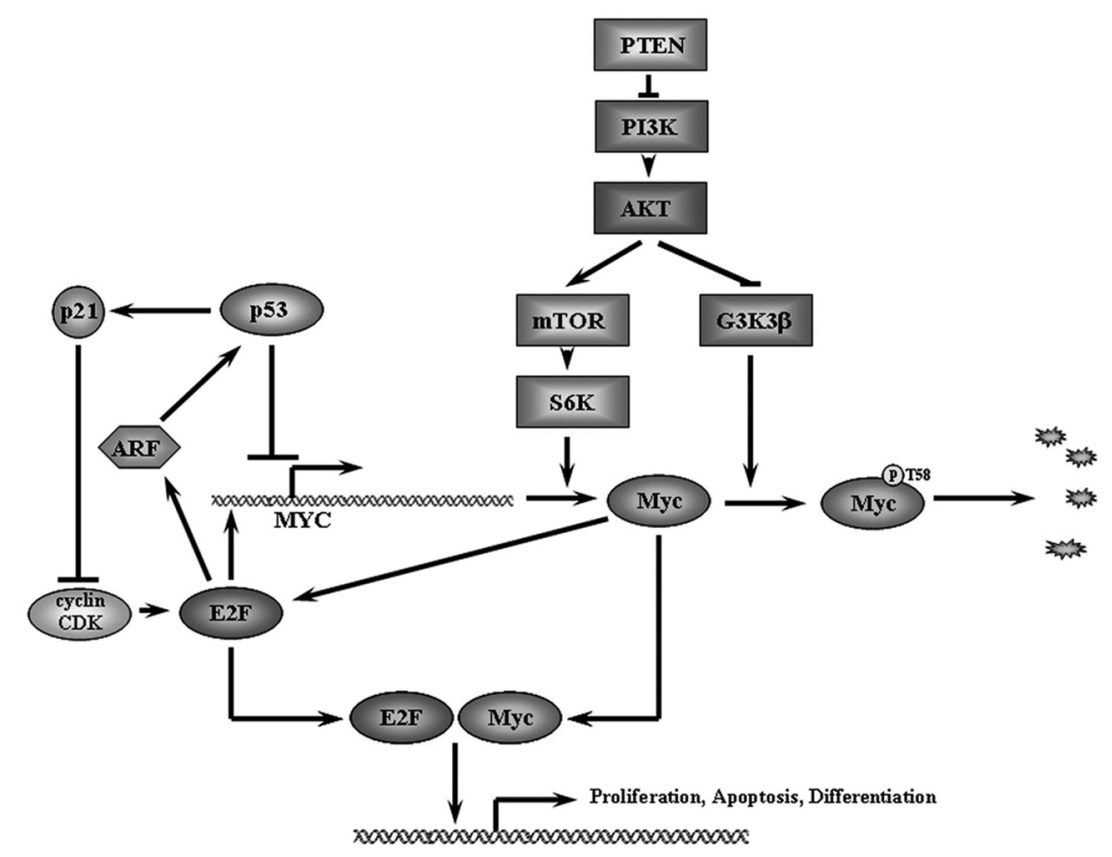

Figure 9. $p 53$ and Pten converge on c-Myc to regulate normal and malignant stem/progenitor self-renewal and differentiation. Combinatorial inactivation of both of the $p 53$ and Pten tumor suppressors collectively elevate c-Myc and E2F activity to enhance NSC self-renewal and impede their differentiation capacity. 
shilfe). Grant support comes from the Goldhirsh Foundation (R.A.D.) and National Institutes of Health grants U01 CA84313 (R.A.D.), RO1CA99041 (L.C.), and 5P01CA95616 (R.A.D., L.C., W.H.W., C.B., and K.L.L.). R.A.D. is an American Cancer Society Research Professor supported by the Robert A. and Renee E. Belfer Foundation Institute for Innovative Cancer Science.

\section{REFERENCES}

Al-Hajj, M., Wicha, M.S., Benito-Hernandez, A., Morrison, S.J., and Clarke, M.F. 2003. Prospective identification of tumorigenic breast cancer cells. Proc. Natl. Acad. Sci. 100: 3983-3988.

Alvarez-Buylla, A., Garcia-Verdugo, J.M., and Tramontin, A.D. 2001. A unified hypothesis on the lineage of neural stem cells. Nat. Rev. Neurosci. 2: 287-293.

Bachoo, R.M., Maher, E.A., Ligon, K.L., Sharpless, N.E., Chan, S.S., You, M.J., Tang, Y., DeFrances, J., Stover, E., Weissleder, R., et al. 2002. Epidermal growth factor receptor and Ink4a/ Arf: Convergent mechanisms governing terminal differentiation and transformation along the neural stem cell to astrocyte axis. Cancer Cell 1: 269-277.

Backman, S.A., Stambolic, V., Suzuki, A., Haight, J., Elia, A., Pretorius, J., Tsao, M.S., Shannon, P., Bolon, B., Ivy, G.O., and Mak, T.W. 2001. Deletion of Pten in mouse brain causes seizures, ataxia and defects in soma size resembling Lhermitte-Duclos disease. Nat. Genet. 29: 396-403.

Bao, S., Wu, Q., McLendon, R.E., Hao, Y., Shi, Q., Hjelmeland, A.B., Dewhirst, M.W., Bigner, D.D., and Rich, J.N. 2006. Glioma stem cells promote radioresistance by preferential activation of the DNA damage response. Nature 444: 756-760.

Ben-Porath, I., Thomson, M.W., Carey, V.J., Ge, R., Bell, G.W., Regev, A., and Weinberg, R.A. 2008. An embryonic stem celllike gene expression signature in poorly differentiated aggressive human tumors. Nat. Genet. 40: 499-507.

Bonnet, D. and Dick, J.E. 1997. Human acute myeloid leukemia is organized as a hierarchy that originates from a primitive hematopoietic cell. Nat. Med. 3: 730-737.

Bredel, M., Bredel, C., Juric, D., Harsh, G.R., Vogel, H., Recht, L.D., and Sikic, B.I. 2005. Functional network analysis reveals extended gliomagenesis pathway maps and three novel MYCinteracting genes in human gliomas. Cancer Res. 65: 86798689.

Calabrese, C., Poppleton, H., Kocak, M., Hogg, T.L., Fuller, C., Hamner, B., Oh, E.Y., Gaber, M.W., Finklestein, D., Allen, M., et al. 2007. A perivascular niche for brain tumor stem cells. Cancer Cell 11: 69-82.

Cartwright, P., McLean, C., Sheppard, A., Rivett, D., Jones, K., and Dalton, S. 2005. LIF/STAT3 controls ES cell self-renewal and pluripotency by a Myc-dependent mechanism. Development 132: 885-896.

Chang, C.J., Mulholland, D.J., Valamehr, B., Mosessian, S., Sellers, W.R., and Wu, H. 2008. PTEN nuclear localization is regulated by oxidative stress and mediates p53-dependent tumor suppression. Mol. Cell. Biol. 28: 3281-3289.

Clarke, M.F. and Fuller, M. 2006. Stem cells and cancer: Two faces of eve. Cell 124: 1111-1115.

Clarke, M.F., Dick, J.E., Dirks, P.B., Eaves, C.J., Jamieson, C.H., Jones, D.L., Visvader, J., Weissman, I.L., and Wahl, G.M. 2006. Cancer stem cells-Perspectives on current status and future directions: AACR Workshop on cancer stem cells. Cancer Res. 66: 9339-9344.

Coppola, J.A. and Cole, M.D. 1986. Constitutive c-myc oncogene expression blocks mouse erythroleukaemia cell differentiation but not commitment. Nature 320: 760-763.

Doetsch, F., Petreanu, L., Caille, I., Garcia-Verdugo, J.M., and Alvarez-Buylla, A. 2002. EGF converts transit-amplifying neurogenic precursors in the adult brain into multipotent stem cells. Neuron 36: 1021-1034.

Fomchenko, E.I. and Holland, E.C. 2006. Mouse models of brain tumors and their applications in preclinical trials. Clin. Cancer Res. 12: 5288-5297.
Fukushima, T., Favereaux, A., Huang, H., Shimizu, T., Yonekawa, Y., Nakazato, Y., and Ohagki, H. 2006. Genetic alterations in primary glioblastomas in Japan. J. Neuropathol. Exp. Neurol. 65: $12-18$.

Furnari, F.B., Fenton, T., Bachoo, R.M., Mukasa, A., Stommel, J.M., Stegh, A., Hahn, W.C., Ligon, K.L., Louis, D.N., Brennan, C., et al. 2007. Malignant astrocytic glioma: Genetics, biology, and paths to treatment. Genes Dev. 21: 2683-2710.

Gage, F.H. 2000. Mammalian neural stem cells. Science 287: $1433-1438$.

Gera, J.F., Mellinghoff, I.K., Shi, Y., Rettig, M.B., Tran, C., Hsu, J.H., Sawyers, C.L., and Lichtenstein, A.K. 2004. AKT activity determines sensitivity to mammalian target of rapamycin (mTOR) inhibitors by regulating cyclin D1 and c-myc expression. J. Biol. Chem. 279: 2737-2746.

Gilbertson, R.J. and Gutmann, D.H. 2007. Tumorigenesis in the brain: Location, location, location. Cancer Res. 67: 55795582.

GilPerotin, S., Marin-Husstege, M., Li, J., Soriano-Navarro, M., Zindy, F., Roussel, M.F., Garcia-Verdugo, J.M., and CasacciaBonnefil, P. 2006. Loss of p53 induces changes in the behavior of subventricular zone cells: Implication for the genesis of glial tumors. J. Neurosci. 26: 1107-1116.

Groszer, M., Erickson, R., Scripture-Adams, D.D., Dougherty, J.D., Le Belle, J., Zack, J.A., Geschwind, D.H., Liu, X., Kornblum, H.I., and Wu, H. 2006. PTEN negatively regulates neural stem cell self-renewal by modulating $\mathrm{G}_{0}-\mathrm{G}_{1}$ cell cycle entry. Proc. Natl. Acad. Sci. 103: 111-116.

Ho, J.S., Ma, W., Mao, D.Y., and Benchimol, S. 2005. p53Dependent transcriptional repression of c-myc is required for $\mathrm{G}_{1}$ cell cycle arrest. Mol. Cell. Biol. 25: 7423-7431.

Jaenisch, R. and Young, R. 2008. Stem cells, the molecular circuitry of pluripotency and nuclear reprogramming. Cell 132: $567-582$.

Kleihues, P. and Ohgaki, H. 1999. Primary and secondary glioblastomas: From concept to clinical diagnosis. NeuroOncology 1: 44-51.

Knoepfler, P.S., Zhang, X.Y., Cheng, P.F., Gafken, P.R., McMahon, S.B., and Eisenman, R.N. 2006. Myc influences global chromatin structure. EMBO J. 25: 2723-2734.

Kwon, C.H., Zhu, X., Zhang, J., Knoop, L.L., Tharp, R., Smeyne, R.J., Eberhart, C.G., Burger, P.C., and Baker, S.J. 2001. Pten regulates neuronal soma size: A mouse model of LhermitteDuclos disease. Nat. Genet. 29: 404-411.

Lassman, A.B., Dai, C., Fuller, G.N., Vickers, A.J., and Holland, E.C. 2004. Overexpression of c-MYC promotes an undifferentiated phenotype in cultured astrocytes and allows elevated Ras and Akt signaling to induce gliomas from GFAP-expressing cells in mice. Neuron Glia Biol. 1: 157-163.

Lee, J., Kotliarova, S., Kotliarov, Y., Li, A., Su, Q., Donin, N.M., Pastorino, S., Purow, B.W., Christopher, N., Zhang, W., Park, J.K., and Fine, H.A. 2006. Tumor stem cells derived from glioblastomas cultured in bFGF and EGF more closely mirror the phenotype and genotype of primary tumors than do serumcultured cell lines. Cancer Cell 9: 391-403.

Libermann, T.A., Nusbaum, H.R., Razon, N., Kris, R., Lax, I., Soreq, H., Whittle, N., Waterfield, M.D., Ullrich, A., and Schlessinger, J. 1985. Amplification, enhanced expression and possible rearrangement of EGF receptor gene in primary human brain tumours of glial origin. Nature 313: 144-147.

Ligon, K.L., Huillard, E., Mehta, S., Kesari, S., Liu, H., Alberta, J.A., Bachoo, R.M., Kane, M., Louis, D.N., DePinho, R.A., et al. 2007. Olig2-regulated lineage-restricted pathway controls replication competence in neural stem cells and malignant glioma. Neuron 53: 503-517.

Lobo, N.A., Shimono, Y., Qian, D., and Clarke, M.F. 2007. The biology of cancer stem cells. Annu. Rev. Cell Dev. Biol. 23: 675-699.

Louis, D.N. 2006. Molecular pathology of malignant gliomas. Annu. Rev. Pathol. 1: 97-117.

Louis, D.N., Ohgaki, H., Wiestler, O.D., and Cavenee, W.K. 2007. WHO classification of tumours of the central nervous system, 4th ed. World Health Organization, Lyon, France.

Meletis, K., Wirta, V., Hede, S.M., Nister, M., Lundeberg, J., and 
Frisen, J. 2006. p53 suppresses the self-renewal of adult neural stem cells. Development 133: 363-369.

Nesbit, C.E., Tersak, J.M., and Prochownik, E.V. 1999. MYC oncogenes and human neoplastic disease. Oncogene 18: 3004-3016.

O’Brien, C.A., Pollett, A., Gallinger, S., and Dick, J.E. 2007. A human colon cancer cell capable of initiating tumour growth in immunodeficient mice. Nature 445: 106-110.

Ohgaki, H., Dessen, P., Jourde, B., Horstmann, S., Nishikawa, T., Di Patre, P.L., Burkhard, C., Schuler, D., Probst-Hensch, N.M., Maiorka, P.C., et al. 2004. Genetic pathways to glioblastoma: A population-based study. Cancer Res. 64: 6892-6899.

Patel, J.H., Loboda, A.P., Showe, M.K., Showe, L.C., and McMahon, S.B. 2004. Analysis of genomic targets reveals complex functions of MYC. Nat. Rev. Cancer 4: 562-568.

Pelengaris, S., Khan, M., and Evan, G. 2002. c-MYC: More than just a matter of life and death. Nat. Rev. Cancer 2: 764-776.

Phillips, H.S., Kharbanda, S., Chen, R., Forrest, W.F., Soriano, R.H., Wu, T.D., Misra, A., Nigro, J.M., Colman, H., Soroceanu, L., et al. 2006. Molecular subclasses of high-grade glioma predict prognosis, delineate a pattern of disease progression, and resemble stages in neurogenesis. Cancer Cell 9: 157-173.

Reardon, D.A., Rich, J.N., Friedman, H.S., and Bigner, D.D. 2006. Recent advances in the treatment of malignant astrocytoma. J. Clin. Oncol. 24: 1253-1265.

Reya, T., Morrison, S.J., Clarke, M.F., and Weissman, I.L. 2001 Stem cells, cancer, and cancer stem cells. Nature 414: 105-111.

Ricci-Vitiani, L., Lombardi, D.G., Pilozzi, E., Biffoni, M., Todaro, M., Peschle, C., and De Maria, R. 2007. Identification and expansion of human colon-cancer-initiating cells. Nature 445: $111-115$.

Sanai, N., Alvarez-Buylla, A., and Berger, M.S. 2005. Neural stem cells and the origin of gliomas. N. Engl. J. Med. 353: 811-822.

Sears, R., Nuckolls, F., Haura, E., Taya, Y., Tamai, K., and Nevins, J.R. 2000. Multiple Ras-dependent phosphorylation pathways regulate Myc protein stability. Genes Dev. 14: 2501-2514.

Shachaf, C.M., Kopelman, A.M., Arvanitis, C., Karlsson, A., Beer, S., Mandl, S., Bachmann, M.H., Borowsky, A.D., Ruebner, B., Cardiff, R.D., et al. 2004. MYC inactivation uncovers pluripotent differentiation and tumour dormancy in hepatocellular cancer. Nature 431: 1112-1117.

Singh, S.K., Clarke, I.D., Hide, T., and Dirks, P.B. 2004a. Cancer stem cells in nervous system tumors. Oncogene 23: 7267-7273.

Singh, S.K., Hawkins, C., Clarke, I.D., Squire, J.A., Bayani, J., Hide, T., Henkelman, R.M., Cusimano, M.D., and Dirks, P.B. 2004b. Identification of human brain tumour initiating cells. Nature 432: 396-401.

Stommel, J.M., Kimmelman, A.C., Ying, H., Nabioullin, R., Ponugoti, A.H., Wiedemeyer, R., Stegh, A.H., Bradner, J.E., Ligon, K.L., Brennan, C., DePinho, R.A., and Chin, L. 2007. Coactivation of receptor tyrosine kinases affects the response of tumor cells to targeted therapies. Science 318: 287-290.

Takahashi, K. and Yamanaka, S. 2006. Induction of pluripotent stem cells from mouse embryonic and adult fibroblast cultures by defined factors. Cell 126: 663-676.

Taylor, M.D., Poppleton, H., Fuller, C., Su, X., Liu, Y., Jensen, P., Magdaleno, S., Dalton, J., Calabrese, C., Board, J., et al. 2005. Radial glia cells are candidate stem cells of ependymoma. Cancer Cell 8: 323-335.

Temple, S. 2001. The development of neural stem cells. Nature 414: $112-117$

Tothova, Z., Kollipara, R., Huntly, B.J., Lee, B.H., Castrillon, D.H., Cullen, D.E., McDowell, E.P., Lazo-Kallanian, S., Williams, I.R., Sears, C., et al. 2007. FoxOs are critical mediators of hematopoietic stem cell resistance to physiologic oxidative stress. Cell 128: 325-339.

Watanabe, K., Tachibana, O., Sata, K., Yonekawa, Y., Kleihues, P., and Ohgaki, H. 1996. Overexpression of the EGF receptor and p53 mutations are mutually exclusive in the evolution of primary and secondary glioblastomas. Brain Pathol. 6: 217-224.

Wiedemeyer, R., Brennan, C., Heffernan, T.P., Xiao, Y., Mahoney, J., Protopopov, A., Zheng, H., Bignell, G., Furnari, F., Cavenee, W.K., et al. 2008. Feedback circuit among INK4 tumor suppressors constrains human glioblastoma development. Cancer Cell 13: 355-364.

Wong, D.J., Liu, H., Ridky, T.W., Cassarino, D., Segal, E., and Chang, H.Y. 2008. Module map of stem cell genes guides creation of epithelial cancer stem cells. Cell Stem Cell 2: 333-344.

Yang, L., Dan, H.C., Sun, M., Liu, Q., Sun, X.M., Feldman, R.I., Hamilton, A.D., Polokoff, M., Nicosia, S.V., Herlyn, M., Sebti, S.M., and Cheng, J.Q. 2004. Akt/protein kinase B signaling inhibitor-2, a selective small molecule inhibitor of Akt signaling with antitumor activity in cancer cells overexpressing Akt. Cancer Res. 64: 4394-4399.

Yilmaz, O.H., Valdez, R., Theisen, B.K., Guo, W., Ferguson, D.O., Wu, H., and Morrison, S.J. 2006. Pten dependence distinguishes haematopoietic stem cells from leukaemia-initiating cells. Nature 441: 475-482.

Zhang, J., Grindley, J.C., Yin, T., Jayasinghe, S., He, X.C., Ross, J.T., Haug, J.S., Rupp, D., Porter-Westpfahl, K.S., Wiedemann, L.M., Wu, H., and Li, L. 2006. PTEN maintains haematopoietic stem cells and acts in lineage choice and leukaemia prevention. Nature 441: 518-522.

Zheng, H., Ying, H., Yan, H., Kimmelman, A.C., Hiller, D.J., Chen, A.-J., Perry, S.R., Tonon, G., Chu, G.C., Ding, Z., et al. 2008. p53 and Pten control neural and glioma stem/progenitor cell renewal and differentiation. Nature 455: 1129-1133.

Zhu, Y. and Parada, L.F. 2002. The molecular and genetic basis of neurological tumours. Nat. Rev. Cancer 2: 616-626.

Zhu, Y., Guignard, F., Zhao, D., Liu, L., Burns, D.K., Mason, R.P., Messing, A., and Parada, L.F. 2005. Early inactivation of p53 tumor suppressor gene cooperating with NF1 loss induces malignant astrocytoma. Cancer Cell 8: 119-130.

Zhuo, L., Theis, M., Alvarez-Maya, I., Brenner, M., Willecke, K., and Messing, A. 2001. hGFAP-cre transgenic mice for manipulation of glial and neuronal function in vivo. Genesis 31: 85-94. 


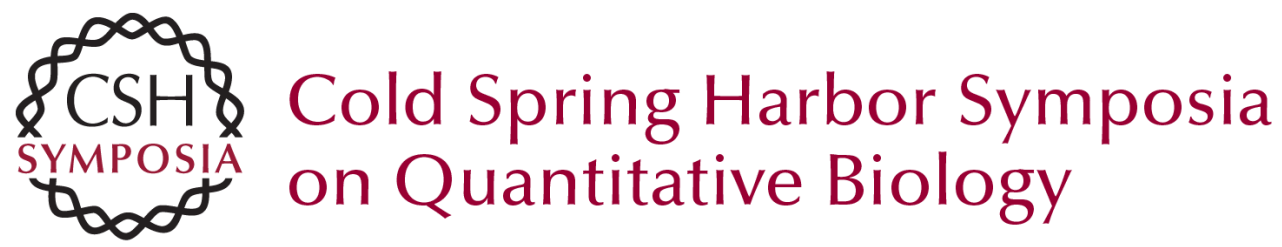

\title{
Pten and p53 Converge on c-Myc to Control Differentiation, Self-renewal, and Transformation of Normal and Neoplastic Stem Cells in Glioblastoma
}

\author{
H. Zheng, H. Ying, H. Yan, et al.
}

Cold Spring Harb Symp Quant Biol 2008 73: 427-437 originally published online January 15, 2009 Access the most recent version at doi:10.1101/sqb.2008.73.047

References This article cites 64 articles, 20 of which can be accessed free at: http://symposium.cshlp.org/content/73/427.full.html\#ref-list-1

\section{License}

Email Alerting Receive free email alerts when new articles cite this article - sign up in the box at the Service top right corner of the article or click here.

To subscribe to Cold Spring Harbor Symposia on Quantitative Biology go to: http://symposium.cshlp.org/subscriptions 